ISSN 0103-5150

Fisioter. Mov., Curitiba, v. 25, n. 1, p. 207-217, jan./mar. 2012 Licenciado sob uma Licença Creative Commons

\title{
0 ultrassom é efetivo no tratamento da tendinite calcárea do ombro?
}

\author{
Ultrasound is effective in treating calcifying \\ tendinitis of the shoulder?
}

Josiane Sena dos Santos ${ }^{[a]}$, Rebecca Barbosa de Decco Monteiro Marinho ${ }^{[b]}$, Angélica Rodrigues Araújo ${ }^{[\mathrm{cl}}$, Maria Emília de Abreu Chaves ${ }^{[\mathrm{d}]}$, Mariane Palumbo Nascimento ${ }^{[\mathrm{e}]}$, Aline de Morais Pereira ${ }^{[\mathrm{f}]}$

[a] Acadêmica de Fisioterapia, Pontifícia Universidade Católica de Minas Gerais (PUC Minas), Belo Horizonte, MG - Brasil, e-mail: josy.sena@yahoo.com.br

[b] Acadêmica de Fisioterapia, Pontifícia Universidade Católica de Minas Gerais (PUC Minas), Belo Horizonte, MG - Brasil, e-mail: beca_monteiro@yahoo.com.br

[c] Fisioterapeuta, Doutora em Bioengenharia pela Universidade Federal de Minas Gerais (UFMG), docente do curso de Fisioterapia da PUC Minas, Belo Horizonte, MG - Brasil, e-mail: angelica@bios.srv.br

[d] Fisioterapeuta, Especialista em Fisioterapia Ortopédica e Esportiva pela Universidade Federal de Minas Gerais (UFMG), Belo Horizonte, MG - Brasil, e-mail: mariaemiliabh@yahoo.com.br

[e] Fisioterapeuta, Especialista em Geriatria e Gerontologia pela Universidade Federal de Minas Gerais (UFMG), Belo Horizonte, MG - Brasil, e-mail: marianepalumbo@yahoo.com.br

[f] Fisioterapeuta, Especialista em Fisioterapia Ortopédica e Desportiva pela Faculdade de Ciências Médicas de Minas Gerais (FCMMG), Belo Horizonte, MG - Brasil, e-mail: alinedemorais@yahoo.com.br

\section{Resumo}

Introdução: A tendinite calcária do ombro é uma calcificação caracterizada pela deposição de sais de cálcio nos tendões do manguito rotador, provocando dor no ombro e perda de amplitude de movimento (ADM). O ultrassom é sugerido como alternativa promissora para facilitar a reabsorção dos depósitos de cálcio. Os mecanismos que fundamentam esse efeito são, entretanto, ainda controversos na literatura, e a utilização clínica do ultrassom na tendinite calcária tem sido baseada mais em evidências empíricas do que científicas. Objetivos: Realizar uma revisão bibliográfica sobre os efeitos biológicos e terapêuticos do ultrassom, a fim de identificar o real papel desse recurso no tratamento da tendinite calcária do ombro. Materiais e métodos: Para sua elaboração, foi feita uma busca bibliográfica em bases de dados eletrônicas e uma pesquisa 
manual. Resultados: Dentre os estudos selecionados, dois demonstraram redução da dor e do tamanho da calcificação e dois não apresentaram resultados positivos. Discussão: Os benefícios promovidos pelo ultrassom são dependentes dos parâmetros utilizados. Variáveis como o tamanho da área a ser tratada, diferenças teciduais, duração da aplicação e o objetivo da conduta terapêutica também devem ser considerados. Conclusão: Com base nesta revisão, pode-se afirmar que o ultrassom terapêutico é um recurso efetivo no tratamento da tendinite calcária do ombro.

Palavras-chave: Terapia por ultrassom. Tendão. Calcificação patológica. Fonoforese.

\section{Abstract}

Introduction: The calcifying tendinitis of the shoulder is a calcification characterized by calcium deposit in the rotator cuff tendons, causing pain and loss of shoulder range of motion (ROM). The ultrasound is suggested as a promising alternative to facilitate the reabsorption of calcium deposits. The mechanisms underlying this effect are still controversial in literature and the clinical use of ultrasound in the calcifying tendinitis has been based more on empirical evidence than scientific. Objectives: To review the ultrasound biological and therapeutic effects for the purpose to identify the true role of this resource in the treatment of calcifying tendinitis of the shoulder. Materials and methods: It made a literature search in electronic databases and a manual research. Results: Among the selected studies, two demonstrated pain and size of the calcification reduction and two studies not showed positive results. Discussion: The benefits promoted by ultrasound are dependent on parameters used. Variables such as the size of treated area, tissue differences, duration of application and therapeutic aim should also be considered. Conclusion: Based in this review, it can be affirmed that ultrasound therapy is an effective resource in the treatment of calcifying shoulder tendinitis.

Keywords: Ultrasonic therapy. Tendon. Calcinosis. Phonophoresis.

\section{Introdução}

A tendinite calcária do ombro é uma calcificação reativa (1), autolimitante (2), caracterizada pela deposição de sais de cálcio nos tendões do manguito rotador (principalmente no tendão do supraespinhoso) (3) e por constantes dores no ombro (4). Conforme Speed e Hazleman (2), essa patologia é mais comum em indivíduos entre 30 e 60 anos de idade, com maior incidência no sexo feminino, podendo ser encontrada tanto em indivíduos sintomáticos quanto assintomáticos (5). Em pacientes sintomáticos, a dor e a perda de amplitude de movimento (ADM) representam as principais queixas (6). A tendinite calcária do ombro corresponde a 17\% das síndromes dolorosas do ombro (7).

A razão para a deposição de cálcio no manguito rotador ainda não é bem esclarecida (2). Fatores como relativa isquemia em consequência da hipovascularização na chamada zona crítica do manguito rotador (8), degeneração dos tendões (9) e distúrbios metabólicos (10) têm sido sugeridos como possíveis causas.
Segundo a literatura, a deposição de cálcio nos tendões ocorre de forma espontânea, podendo haver somente uma fase aguda, em que os sintomas tendem a diminuir em poucas semanas, ou a evolução para a cronicidade (11). Nesse caso, prejuízos funcionais, em razão, principalmente, da dor, da diminuição da amplitude de movimento $(6,11)$ e das alterações na função mecânica do ombro $(11,12)$ são comumente observados. Talvez, por essas razões, Uhthoff e Sarkar $(7,13)$ consideram a tendinite calcária do ombro como condição incapacitante.

A abordagem terapêutica da tendinite calcária do ombro consiste na utilização de anti-inflamatórios não esteroides $(4,14)$, infiltração com corticoide (14), punção (4) e terapia por ondas de choque extracorpórea (4). Aspiração percutânea (4, 15, 16) e cirurgia (11) também são procedimentos realizados, porém somente em último caso, em razão do caráter invasivo de ambas. Os efeitos desses tratamentos variam significantemente e os resultados são, na maioria das vezes, inconsistentes (17). 
Dentre os recursos fisioterapêuticos utilizados para o tratamento da tendinite calcária do ombro, o ultrassom tem sido sugerido como alternativa promissora para facilitar a reabsorção dos depósitos de cálcio $(15,18-21)$. Os mecanismos que fundamentam esse efeito são, entretanto, ainda controversos na literatura e a utilização clínica do ultrassom na tendinite calcária tem sido baseada mais em evidências empíricas do que científicas. Este estudo objetivou realizar uma revisão bibliográfica sobre os efeitos biológicos e terapêuticos do ultrassom, a fim de identificar o seu real papel no tratamento de depósitos calcários, especificamente na tendinite calcária do ombro.

\section{Metodologia}

\section{Busca literária}

Para a realização desta pesquisa bibliográfica foram consultadas as seguintes bases de dados: MEDLINE (1966 até abril de 2010), PubMed (1966 até abril de 2010), Cochrane Library (1991 até abril de 2010), PEDro (até abril de 2010) e LILACS (1982 até abril de 2010). Os descritores utilizados foram: calcifying tendinitis, calcific tendinitis, calcium deposits, tendinitis calcarea, paratentinitis calcarea, calcification tendons, calcareous tendinitis, ultrasound therapy, therapeutic ultrasound, phonophoresis. Esses termos foram combinados entre si ou aplicados de forma isolada nas bases de dados, como pode ser visto na Tabela 1. Estudos aleatorizados ou não, controlados e publicados na língua portuguesa, inglesa ou espanhola foram incluídos.

\section{Outras estratégias de busca}

Além das bases eletrônicas, a busca foi complementada por uma pesquisa manual nas referências bibliográficas dos artigos previamente selecionados e no acervo da biblioteca da Pontifícia Universidade Católica de Minas Gerais.

\section{Seleção dos estudos}

Dois revisores independentes selecionaram inicialmente os artigos, de acordo com o título e o resumo.
Tabela 1 - Protocolo utilizado para a busca literária nas base de dados MEDLINE, PubMed, Cochrane, PEDro e LILACS

(Continua)

\begin{tabular}{|c|c|}
\hline Base de dados & Palavras-chave \\
\hline $\begin{array}{l}\text { MEDLINE, PubMed, } \\
\text { Cochrane }\end{array}$ & $\begin{array}{l}\text { 1. phonophoresis } \\
\text { 2. ultrasound therapy } \\
\text { 3. therapeutic ultrasound } \\
\text { 4. transdermal drug application } \\
\text { 5. transdermal drug delivery } \\
\text { 6. } 1 \text { or } 2 \text { or } 3 \text { or } 4 \text { or } 5 \\
\text { 7. tendinitis calcarea } \\
\text { 8. paratendinitis calcarea } \\
\text { 9. calcification tendons } \\
\text { 10. calcareous tendinitis } \\
\text { 11. calcifying tendinitis } \\
\text { 12. calcific tendinitis } \\
\text { 13. calcium deposits } \\
\text { 14. pain } \\
\text { 15. } 7 \text { or } 8 \text { or } 9 \text { or } 10 \text { or } 11 \text { or } 12 \text { or } \\
13 \text { or } 14 \\
\text { 16. } 6 \text { AND } 15\end{array}$ \\
\hline PEDro, LILACS & $\begin{array}{l}\text { 1. phonophoresis AND tendinitis } \\
\text { calcarea } \\
\text { 2. phonophoresis AND paratendinitis } \\
\text { calcarea } \\
\text { 3. phonophoresis AND calcification } \\
\text { tendons } \\
\text { 4. phonophoresis AND calcareous } \\
\text { tendinitis } \\
\text { 5. phonophoresis AND calcifying } \\
\text { tendinitis } \\
\text { 6. phonophoresis AND calcific } \\
\text { tendinitis } \\
\text { 7. phonophoresis AND calcium } \\
\text { deposits } \\
\text { 8. phonophoresis AND pain } \\
\text { 9. ultrasound therapy AND tendinitis } \\
\text { calcarea } \\
\text { 10. ultrasound therapy AND } \\
\text { paratendinitis calcarea } \\
\text { 11. ultrasound therapy AND } \\
\text { calcification tendons } \\
\text { 12. ultrasound therapy AND } \\
\text { calcareous tendinitis } \\
\text { 13. ultrasound therapy AND calcifying } \\
\text { tendinitis } \\
\text { 14. ultrasound therapy AND calcific } \\
\text { tendinitis } \\
\text { 15. ultrasound therapy AND calcium } \\
\text { deposits } \\
\text { 16. ultrasound therapy AND calcifying } \\
\text { tendinitis } \\
\text { 17. ultrasound therapy AND calcific } \\
\text { tendinitis } \\
\text { 18. ultrasound therapy AND calcifying } \\
\text { tendinitis }\end{array}$ \\
\hline
\end{tabular}


Tabela 1 - Protocolo utilizado para a busca literária nas base de dados MEDLINE, PubMed, Cochrane, PEDro e LILACS

(Conclusão)

\begin{tabular}{l}
\hline Base de dados \\
PEDro, LILACS
\end{tabular} \begin{tabular}{l} 
19. ultrasound therapy AND calcifying \\
tendinitis \\
20. ultrasound therapy AND calcific \\
tendinitis \\
21. ultrasound therapy AND calcium \\
deposits \\
22. ultrasound therapy AND pain \\
23. transdermal drug application AND \\
tendinitis calcarea \\
24. transdermal drug application AND \\
paratendinitis calcarea \\
25. transdermal drug application AND \\
calcification tendons \\
26. transdermal drug application AND \\
calcareous tendinitis \\
27. transdermal drug application AND \\
calcifying tendinitis \\
28. transdermal drug application AND \\
calcific tendinitis \\
29. transdermal drug application AND \\
calcium deposits \\
30. transdermal drug application AND \\
pain \\
31. transdermal drug delivery AND \\
tendinitis calcarea \\
32. transdermal drug delivery AND \\
paratendinitis calcarea \\
33. transdermal drug delivery AND \\
calcification tendons \\
34. transdermal drug delivery AND \\
calcareous tendinitis \\
35. transdermal drug delivery AND \\
calcifying tendinitis \\
36. transdermal drug delivery AND \\
calcific tendinitis \\
37. transdermal drug delivery AND \\
calcium deposits \\
transdermal drug delivery AND \\
\hline pain
\end{tabular}

Fonte: Dados da pesquisa.

Se tais artigos não forneciam informações suficientes para a inclusão, ou os examinadores verificassem que o artigo era potencialmente útil, uma cópia completa era solicitada. Na presença de divergência entre os avaliadores, quanto à inclusão de algum estudo, estes se reuniam para que um consenso fosse obtido. Não ocorrendo um acordo entre eles, uma terceira pessoa era consultada. Os critérios de exclusão foram artigos em que a amostra compreendia indivíduos com episódio recente de trauma no ombro, artrite do ombro, capsulite adesiva e infecção local.

\section{Desfechos avaliados}

A efetividade da fonoforese foi avaliada por meio de um ou mais dos seguintes desfechos: exame radiológico, amplitude de movimento (ADM), dor, porcentagem da droga transmitida pelo ultrassom e função.

\section{Avaliação da qualidade metodológica}

A análise da qualidade dos artigos selecionados foi realizada de acordo com a escala de Jadad (22). Segundo a escala, as notas variam de 0 a 5 pontos, sendo que pontuação inferior a 3 indica estudo metodologicamente fraco, e entre 3 a 5 indica pesquisa de alta qualidade (23). Dois revisores independentes avaliaram cada artigo utilizando os critérios de Jadad (22). Na presença de não similaridade entre as notas, os dois avaliadores reuniam-se. Permanecendo o desacordo, uma terceira pessoa era consultada.

\section{Análise dos resultados}

As informações referentes ao objetivo, à metodologia e ao desfecho dos artigos selecionados foram organizadas em tabelas, a fim de facilitar a análise e a interpretação de cada estudo. Buscou-se verificar a correlação entre o objetivo e o desfecho do trabalho e avaliar a clareza na determinação e na descrição dos parâmetros utilizados para a pesquisa.

\section{Resultados}

A busca eletrônica inicial identificou 1.108 artigos. Destes, 31 foram selecionados a partir da leitura dos títulos, sendo apenas quatro trabalhos (6, $15,24,25)$ incluídos após a leitura dos resumos (Tabela 2). A exclusão dos 27 estudos ocorreu em razão da utilização do ultrassom não associado a fármaco tópico.

A qualidade metodológica dos estudos variou de 0 a 4 na escala de Jadad ( 0 a 5). Três artigos foram considerados de baixa $(6,24,25)$ e um, de alta (15) qualidade. As falhas metodológicas mais 
Tabela 2 - Resultado da busca literária nas bases de dados MEDLINE, PubMed, Cochrane, PEDro e LILACS

\begin{tabular}{lccc}
\hline $\begin{array}{l}\text { Base de } \\
\text { dados }\end{array}$ & Resultados & $\begin{array}{l}\text { Artigos } \\
\text { selecionados }\end{array}$ & $\begin{array}{l}\text { Artigos } \\
\text { incluídos }\end{array}$ \\
\hline $\begin{array}{l}\text { Ovid } \\
\text { MEDLINE }\end{array}$ & 458 & 5 & 1 \\
$\begin{array}{l}\text { Cochrane } \\
\text { database }\end{array}$ & 430 & 7 & 0 \\
PEDro & 156 & 11 & 2 \\
LILACS & 0 & 0 & 0 \\
PubMed & 64 & 8 & 1 \\
\hline Total & 1108 & 31 & 4 \\
\hline
\end{tabular}

Fonte: Dados da pesquisa.

comuns entre os estudos foram ausência de duplo-cego $(6,24,25)$ e descrição do método de gerar a sequência de aleatorização da amostra $(6,15,24$, 25) (Tabela 3).

Dos quatro trabalhos selecionados $(6,15,24$, $25)$, três $(6,15,24)$ eram estudos clínicos aleatorizados. Os desfechos mais avaliados foram: tamanho da calcificação $(15,24)$, dor $(15,24)$ e $\operatorname{ADM}(6,15,24)$, os quais foram mensurados por meio de raio X e/ou escala Gartner e Heryer, escala visual analógica e goniometria, respectivamente. Dentre os estudos selecionados, dois (15, 24) demonstraram redução da dor e do tamanho da calcificação e dois $(6,25)$ não apresentaram resultados positivos quanto ao uso do ultrassom na tendinite calcária do ombro. Esses dados estão descritos na Tabela 4.

Na Tabela 5, estão discriminados os parâmetros utilizados nos trabalhos. Pode-se observar que a frequência do ultrassom variou de $0,89 \mathrm{MHz}$ a $3 \mathrm{MHz}$, e a intensidade ficou entre $0,8 \mathrm{~W} / \mathrm{cm}^{2}$ a $2,5 \mathrm{~W} / \mathrm{cm}^{2}$, permanecendo ambos dentro da faixa recomendada para aplicações terapêuticas. Em relação ao modo de aplicação, somente um estudo utilizou o ultrassom pulsado (15). 0 tempo de aplicação variou de 5 a 15 minutos e a frequência das sessões foi de 3 a 5 vezes por semana, totalizando de 9 a 24 sessões.

\section{Discussão}

O ultrassom é um recurso amplamente empregado nas afecções do sistema musculoesquelético (26-28), visando principalmente ao controle dos sinais e dos sintomas inflamatórios (29-32), ao estímulo à fibroplasia e à osteogênese $(32,33)$ e à modulação da dor (26). Os benefícios induzidos por esse recurso são decorrentes de suas ações térmicas e não térmicas nos tecidos (26). Tradicionalmente, esses efeitos são considerados separadamente, embora ambos possam ocorrer em todas as aplicações do ultrassom.

Os efeitos térmicos do ultrassom, incluindo aceleração do metabolismo, alteração da velocidade de condução nervosa, aumento do fluxo sanguíneo e da extensibilidade de tecidos moles, redução ou controle da dor e do espasmo muscular $(30,34)$, são os mesmos obtidos com outras modalidades de aquecimento; porém as estruturas-alvo do aquecimento (tecidos ricos em proteínas, principalmente colágeno) são diferentes (35). Os efeitos atérmicos resultam de eventos mecânicos (cavitação, correntes acústicas e microfluxo) produzidos pela passagem da onda sonora nos tecidos e estão relacionados: 1) ao aumento da permeabilidade da pele (36) e da membrana celular (37); 2) ao aumento dos níveis de cálcio intracelular (38); 3) ao aumento da síntese proteica e da atividade de fibroblastos e condrócitos $(39,40)$ e 4$)$ ao aumento da degranulação de mastócitos (41) e da atividade dos macrófagos (42).

Os benefícios supracitados são, entretanto, dependentes dos parâmetros utilizados para aplicação do ultrassom (43), principalmente da dosimetria. Variáveis como o tamanho da área a ser tratada, diferenças teciduais (44), duração da aplicação (43) e o objetivo da conduta terapêutica (44) também devem ser considerados.

Em uma revisão realizada por Gam e Johannsen (17), em que foram analisados artigos publicados entre 1950 e 1992, foi concluído que apenas 22 dos 293 artigos revisados apresentavam metodologia adequada, sendo apenas esses os que comprovaram a eficácia do ultrassom. Além disso, os autores relataram a dificuldade de investigar uma possível relação entre dose/resposta em razão do déficit de informações sobre os parâmetros utilizados na aplicação desse recurso.

De maneira semelhante, no trabalho de Robertson e Baker (19) foi avaliada a qualidade metodológica de estudos que abordavam a efetividade 
Tabela 3 - Pontuação dos estudos na escala de Jadad

\begin{tabular}{|c|c|c|c|c|c|c|c|}
\hline Autor & Randomizado & Duplo-cego & $\begin{array}{l}\text { Descrição de } \\
\text { desistência ou } \\
\text { exclusão da } \\
\text { amostra }\end{array}$ & $\begin{array}{l}\text { Descrição do } \\
\text { método alea- } \\
\text { torização }\end{array}$ & $\begin{array}{l}\text { Descrição do } \\
\text { método de } \\
\text { duplo-cego }\end{array}$ & Nota & $\begin{array}{l}\text { Qualidade } \\
\text { metodoló- } \\
\text { gica }\end{array}$ \\
\hline $\begin{array}{l}\text { Shomoto et al., } \\
2002 \text { (24) }\end{array}$ & 1 & - & 1 & - & - & 2 & Baixa \\
\hline $\begin{array}{l}\text { Ebenbichler et } \\
\text { al., } 1999(15)\end{array}$ & 1 & 1 & 1 & 1 & - & 4 & Alta \\
\hline $\begin{array}{l}\text { Perron e } \\
\text { Malouin, } 1997 \\
\text { (6) }\end{array}$ & 1 & - & 1 & - & - & 2 & Baixa \\
\hline $\begin{array}{l}\text { Greve, } 1991 \\
\text { (25) }\end{array}$ & - & - & - & - & - & 0 & Baixa \\
\hline
\end{tabular}

Fonte: Dados da pesquisa.

Tabela 4 - Caracterização dos estudos selecionados

(Continua)

\begin{tabular}{|c|c|c|c|c|c|}
\hline Autor/ano & $\begin{array}{l}\text { Tipo de } \\
\text { estudo }\end{array}$ & $\begin{array}{l}\text { Objetivo do } \\
\text { estudo }\end{array}$ & $\begin{array}{l}\text { Amostra/grupos de } \\
\text { estudo }\end{array}$ & Desfechos avaliados & Resultados \\
\hline $\begin{array}{l}\text { Shomoto et al., } \\
2002(24)\end{array}$ & $\begin{array}{l}\text { Ensaio clínico } \\
\text { aleatorizado }\end{array}$ & $\begin{array}{l}\text { Avaliar o efeito } \\
\text { terapêutico do } \\
\text { ultrassom na } \\
\text { calcificação, e } \\
\text { a dor durante } 0 \\
\text { movimento ativo }\end{array}$ & $\begin{array}{l}40 \text { indivíduos: } 20 \text { no } \\
\text { grupo contole e } 20 \text { no } \\
\text { grupo experimental }\end{array}$ & $\begin{array}{l}1 \text { - Calcificação (es- } \\
\text { cala Gartner e Heryer, } \\
\text { radiografia) } \\
2 \text { - Dor durante } 0 \\
\text { movimento ativo }\end{array}$ & $\begin{array}{l}\text { Diminuição da dor e da } \\
\text { calcificação em ambos } \\
\text { os grupos (diferença } \\
\text { estatisticamente signi- } \\
\text { ficativa) }\end{array}$ \\
\hline $\begin{array}{l}\text { Ebenbichler et al., } \\
1999(15)\end{array}$ & $\begin{array}{l}\text { Randomizado, } \\
\text { duplo-cego. }\end{array}$ & $\begin{array}{l}\text { Avaliar o efeito do } \\
\text { ultrassom terapêu- } \\
\text { tico pulsado no tra- } \\
\text { tamento da ten- } \\
\text { dinite calcária do } \\
\text { ombro idiopática }\end{array}$ & $\begin{array}{l}54 \text { indivíduos/61 om- } \\
\text { bros: } 32 \text { ombros no } \\
\text { grupo experimental e } \\
29 \text { ombros no grupo } \\
\text { controle }\end{array}$ & $\begin{array}{l}\text { Calcificação (escala } \\
\text { Gartner e Heryer, } \\
\text { radiografia) } \\
\text { 2- Dor (escala visual } \\
\text { analógica) } \\
\text { 3- Qualidade de } \\
\text { vida (escala visual } \\
\text { analógica) }\end{array}$ & $\begin{array}{l}\text { Após seis semanas: } \\
\text { diminuição da calcifica- } \\
\text { ção e da dor, melhora } \\
\text { da qualidade de vida } \\
\text { em ambos os grupos, } \\
\text { sendo mais significati- } \\
\text { vo no grupo experi- } \\
\text { mental. Após nove } \\
\text { meses, a diferença } \\
\text { entre os grupos não foi } \\
\text { significativa }\end{array}$ \\
\hline $\begin{array}{l}\text { Perron e Malouin, } \\
1997 \text { (6) }\end{array}$ & $\begin{array}{l}\text { Ensaio clínico } \\
\text { randomizado }\end{array}$ & $\begin{array}{l}\text { Avaliar o efeito } \\
\text { da iontoforese } \\
\text { (ácido acético) } \\
\text { e do ultrassom } \\
\text { no tratamento da } \\
\text { tendinite calcária } \\
\text { do ombro e de- } \\
\text { terminar a relação } \\
\text { entre o tamanho } \\
\text { da calcificação e a } \\
\text { funcionalidade do } \\
\text { ombro }\end{array}$ & $\begin{array}{l}22 \text { indivíduos: } 11 \text { no } \\
\text { grupo experimental e } \\
10 \text { no grupo controle }\end{array}$ & $\begin{array}{l}\text { 1- Calcificação (radio- } \\
\text { grafia) } \\
2 \text { - ADM passiva de } \\
\text { abdução do ombro } \\
\text { 3- Dor (escala present } \\
\text { pain index) }\end{array}$ & $\begin{array}{l}\text { Não houve diferença } \\
\text { significativa das variá- } \\
\text { veis mensuradas entre } \\
\text { ambos os grupos }\end{array}$ \\
\hline
\end{tabular}


Tabela 4 - Caracterização dos estudos selecionados

(Conclusão)

\begin{tabular}{|c|c|c|c|c|c|}
\hline Autor/ano & $\begin{array}{l}\text { Tipo de } \\
\text { estudo }\end{array}$ & $\begin{array}{l}\text { Objetivo do } \\
\text { estudo }\end{array}$ & $\begin{array}{l}\text { Amostra/grupos de } \\
\text { estudo }\end{array}$ & Desfechos avaliados & Resultados \\
\hline $\begin{array}{l}\text { Greve et al., } \\
1991 \text { (25) }\end{array}$ & Não citado & $\begin{array}{l}\text { Estudar os } \\
\text { resultados a longo } \\
\text { prazo da cinesio- } \\
\text { terapia nas lesões } \\
\text { tendinosas do } \\
\text { manguito rotador } \\
\text { do ombro e quais } \\
\text { os fatores que } \\
\text { interferem nesses } \\
\text { resultados }\end{array}$ & $\begin{array}{l}60 \text { ombros de } 58 \\
\text { pacientes divididos de } \\
\text { acordo com o diag- } \\
\text { nóstico: } 31 \text { ombros } \\
\text { com síndrome do } \\
\text { impacto, } 18 \text { com ten- } \\
\text { dinite calcária, } 5 \text { com } \\
\text { ruptura do manguito, } \\
3 \text { com tendinite bi- } \\
\text { ciptal e } 3 \text { com lesões } \\
\text { múltiplas }\end{array}$ & $\begin{array}{l}\text { Ao fim do tratamento, } \\
11 \text { dos } 18 \text { pacientes } \\
\text { com tendinite calcária } \\
\text { tiveram resultados } \\
\text { insatisfatórios; e no } \\
\text { follow-up, de } 6 \text { a } 36 \\
\text { meses, } 13 \text { indivíduos } \\
\text { não melhoraram. Não } \\
\text { houve diferença entre } \\
\text { os resultados encontra- } \\
\text { dos com as alterações } \\
\text { radiológicas }\end{array}$ & \\
\hline
\end{tabular}

Fonte: Dados da pesquisa.

Tabela 5 - Parâmetros do ultrassom utilizados nos trabalhos selecionados

\begin{tabular}{|c|c|c|c|c|c|c|c|c|}
\hline Autor/ano & $\begin{array}{l}\text { Frequên- } \\
\text { cia }\end{array}$ & Modo & $\begin{array}{l}\text { Intensi- } \\
\text { dade (W/ } \\
\left.\mathrm{cm}^{2}\right)\end{array}$ & $\begin{array}{l}\text { Tempo de } \\
\text { aplicação }\end{array}$ & $\begin{array}{l}\text { Local e } \\
\text { tamanho da } \\
\text { aplicação }\end{array}$ & $\begin{array}{l}\text { ERA cabe- } \\
\text { çote }\end{array}$ & $\begin{array}{l}\text { Número de } \\
\text { sessões }\end{array}$ & $\begin{array}{l}\text { Frequên- } \\
\text { cia das } \\
\text { sessões }\end{array}$ \\
\hline $\begin{array}{l}\text { Shomoto } \\
\text { et al., } 2002 \\
\text { (24) }\end{array}$ & $3 \mathrm{MHz}$ & Contínuo & $\begin{array}{l}1.0 \text { a } 2.0 \\
W / \mathrm{cm}^{2}\end{array}$ & 5 minutos & $\begin{array}{l}\text { Bíceps (ombro } \\
\text { em neutro), infra- } \\
\text { espinhal e supra- } \\
\text { espinhal (ombro } \\
\text { em rotação in- } \\
\text { terna), subesca- } \\
\text { pular (ombro em } \\
\text { rotação externa }\end{array}$ & $\begin{array}{l}5 \mathrm{~cm}^{2} \\
\text { (cabeçote) } \\
\text { e } 4,3 \mathrm{~cm}^{2} \\
\text { (ERA) }\end{array}$ & Não citado & $\begin{array}{l}3 \text { vezes por } \\
\text { semana }\end{array}$ \\
\hline $\begin{array}{l}\text { Ebenbichler } \\
\text { et al., } 1999 \\
\text { (15) }\end{array}$ & $0,89 \mathrm{MHz}$ & $\begin{array}{l}\text { Pulsado } \\
(25 \%)\end{array}$ & $2,5 \mathrm{~W} / \mathrm{cm}^{2}$ & 15 minutos & $\begin{array}{l}\text { Subescapular } \\
\text { (ombro em ab- } \\
\text { dução e rotação } \\
\text { externa), supra- } \\
\text { espinhal e infra- } \\
\text { espinhal (ombro } \\
\text { em rotação } \\
\text { interna) }\end{array}$ & $\begin{array}{l}5 \mathrm{~cm}^{2} \\
\text { (cabeçote) }\end{array}$ & 24 sessões & $\begin{array}{l}5 \text { vezes por } \\
\text { semana (15 } \\
\text { primeiras } \\
\text { sessões) } \\
\text { e } 3 \text { vezes } \\
\text { por semana } \\
\text { (últimas } \\
\text { sessões) }\end{array}$ \\
\hline $\begin{array}{l}\text { Perron e } \\
\text { Malouin, } \\
1997 \text { (6) }\end{array}$ & $1 \mathrm{MHz}$ & Contínuo & $\begin{array}{l}0,8 \mathrm{~W} / \mathrm{cm}^{2} \text {; } \\
\text { corrente } \\
\text { galvânica: } \\
5 \mathrm{mAe} \\
5 \% \text { ácido } \\
\text { acético }\end{array}$ & $\begin{array}{l}5 \text { minutos; } \\
20 \text { minutos }\end{array}$ & Não citado & Não citado & 9 sessões & $\begin{array}{l}3 \text { vezes por } \\
\text { semana }\end{array}$ \\
\hline $\begin{array}{l}\text { Greve et } \\
\text { al., } 1991 \\
(25)\end{array}$ & Não citado & Não citado & Não citado & Não citado & Não citado & Não citado & Não citado & Não citado \\
\hline
\end{tabular}

Fonte: Dados da pesquisa. 
do ultrassom terapêutico em indivíduos que apresentavam dor e/ou lesões musculoesqueléticas. Dentre os trabalhos consultados, a falta de grupo controle, de tratamento padronizado e de critérios de avaliação, bem como a ausência da análise estatística dos resultados, caracterizou os estudos como sendo de baixa qualidade metodológica (19).

Efeitos do ultrassom na tendinite calcária do ombro

Conforme demonstrado pela presente revisão, poucos estudos têm investigado os efeitos e/ou a efetividade do ultrassom $(6,15,24,25)$ no tratamento da tendinite calcária do ombro, e os resultados encontrados nos trabalhos selecionados foram variáveis. Apesar disso, a utilização do ultrassom no manejo dessa afecção é trivial na prática clínica do fisioterapeuta $(26,28)$.

Resultados positivos foram observados no trabalho conduzido por Ebenbichler et al. (15), que, por meio de um estudo randomizado e duplo-cego, analisaram a efetividade do ultrassom terapêutico comparado ao placebo no tratamento da tendinite calcária do ombro. Os pacientes do grupo experimental receberam 15 minutos de ultrassom pulsado (1:4) com frequência de 0,89 $\mathrm{MHz}$ e intensidade de $2,5 \mathrm{~W} / \mathrm{cm}^{2}$, com a área do cabeçote de $5 \mathrm{~cm}^{2}$. No grupo controle, o recurso terapêutico foi aplicado pelo mesmo período de tempo, porém desligado. Foram realizadas ao todo 24 sessões. Após seis semanas de intervenção, verificou-se significativa diferença entre os grupos na diminuição do depósito calcário, favorecendo o grupo experimental. Houve redução da dor e melhora da qualidade de vida nesse grupo em relação ao controle após o término do tratamento. No entanto, após os nove meses de follow-up não foi encontrada diferença significativa entre os grupos.

Shomoto et al. (24) realizaram um estudo com 40 pacientes estratificadamente randomizados em dois grupos. 0 grupo experimental $(n=20)$ foi submetido a cinco minutos de ultrassom terapêutico (modo contínuo; $3 \mathrm{MHz} ; 1$ a $2 \mathrm{~W} / \mathrm{cm}^{2}$; ERA de $4,3 \mathrm{~cm}^{2}$; área da superfície de $5 \mathrm{~cm}^{2}$ ) associado a exercícios. 0 grupo controle $(n=20)$ foi tratado apenas com exercícios. Todos os participantes sofreram intervenção três vezes por semana até o final do estudo, porém os autores não informaram a duração da pesquisa. Observou-se, no grupo experimental, melhora significativa da calcificação com poucos pacientes ainda presenciando dor durante a movimentação ativa. No grupo controle, a maioria das calcificações não modificou a área e nem a densidade, ou os pacientes apresentaram piora da condição.

Especificamente em relação às ações do ultrassom na tendinite calcária, Ebenbichler et al. (15) sugerem que os efeitos térmicos induzidos por esse recurso seriam os responsáveis pela redução da calcificação. Os autores afirmam que a atividade das células capazes de fagocitar as partículas de cálcio, assim como o metabolismo e o fluxo sanguíneo, é favorecida pelo aumento da temperatura dos tecidos. Esses efeitos, em conjunto, possibilitariam a desintegração dos depósitos de cálcio. Shomoto et al. (24) acreditam que a redução do depósito calcário também esteja relacionada ao aumento da circulação sanguínea, da atividade dos fagócitos e à liberação de fatores quimiotáticos.

Os parâmetros utilizados por Ebenbichler et al. (15) e Shomoto et al. (24) foram diferentes entre si; no entanto, os resultados encontrados nos dois estudos foram semelhantes, havendo redução da dor e da calcificação. Esses fatos podem ser justificados pela alta dose total de energia fornecida aos pacientes submetidos ao tratamento em ambos os estudos.

Dois estudos $(6,25)$ encontraram resultados divergentes aos trabalhos de Ebenbichler et al. (15) e Shomoto et al. (24).

Perron e Malouin (6) investigaram a efetividade da iontoforese associada ao ultrassom terapêutico na tendinite calcária do ombro de 22 voluntários. 0 grupo experimental foi submetido a nove sessões de iontoforese seguidas de ultrassom contínuo (1 $\mathrm{MHz} ; 0,8 \mathrm{~W} / \mathrm{cm}^{2} ; 5$ minutos). 0 grupo controle não recebeu nenhum tratamento durante o mesmo período. Os autores não encontraram diferença significativa entre os grupos na redução da área e da densidade do depósito calcáreo. 0 mesmo foi observado para dor e abdução passiva do ombro. Concluiu-se que a iontoforese associada ao ultrassom não é efetiva para a reabsorção do depósito calcário, como também na melhora da funcionalidade. A diminuição em ambos os grupos do tamanho e da densidade do depósito calcário resulta, provavelmente, de um processo natural e não do tratamento empregado (6). 0 fato dos autores não terem identificado efeitos positivos no grupo experimental em relação ao controle pode ser em razão da falha metodológica do trabalho.

No estudo de Greve et al. (25), foi utilizado o ultrassom terapêutico e o fortalecimento muscular 
em 60 ombros com disfunção tendinosa. Destes, 18 apresentavam tendinite calcária do ombro. Os parâmetros utilizados para o ultrassom terapêutico não foram mencionados pelos autores e o estudo não apresentava grupo controle. Os pacientes foram tratados duas vezes por semana, de 1 a 8 meses. Ao final do tratamento, 11 dos 18 pacientes tiveram resultados insatisfatórios; e no follow-up, de 6 a 36 meses, 13 indivíduos não melhoraram. Não houve diferença entre os resultados encontrados com as alterações radiológicas. Como os autores não informaram os parâmetros utilizados e não compararam os resultados obtidos com grupo controle, inviabiliza-se afirmar que o ultrassom não promove benefícios para os indivíduos com tendinite calcária do ombro $(19,44)$.

A comparação entre os achados positivos e negativos $(6,15,24,25)$ deve ser feita com cautela, uma vez que os parâmetros são diferentes e, em alguns casos, não são citados $(19,44)$.

A frequência $(\mathrm{MHz})$ do ultrassom é responsável por determinar a profundidade de penetração da onda mecânica no tecido-alvo (39). A literatura afirma que quanto maior a frequência da onda ultrassônica, menor será sua penetração nos tecidos e maior será a absorção $(45,46)$. Considerando que a patologia a ser tratada é de caráter superficial, não seria necessária a utilização de altas frequências. No entanto, resultados positivos e negativos foram observados independentes da frequência.

Assim como a frequência, a intensidade $\left(\mathrm{W} / \mathrm{cm}^{2}\right)$, que influencia nos mecanismos térmicos e atérmicos promovidos pelo ultrassom, também foi variável. De acordo com Low e Reed (47), a energia sonora é convertida em energia térmica, sendo esta proporcional à intensidade do ultrassom. Em todos os estudos avaliados, a intensidade foi aplicada dentro da faixa terapêutica e demonstrou proporcionar efeitos positivos. Portanto, fica claro que a aplicação inadequada desse recurso pode não induzir aos benefícios terapêuticos e/ou causar danos aos tecidos biológicos (25). Por isso, é importante que sejam conhecidos os parâmetros apropriados, a fim de atingir os objetivos propostos, dentro dos limites seguros (48).

As divergências encontradas nos estudos que demonstraram efeitos positivos e negativos podem ser justificadas pelos diferentes parâmetros utilizados nos trabalhos. Apesar de inicialmente os parâmetros usados por Ebenbichler et al. (15) e Shomoto et al. (24) parecerem diferentes, a dose média aplicada por sessão foi semelhante ( $\mathrm{w} / \mathrm{cm}^{2} \mathrm{x}$ minuto).
Nos estudos com resultados positivos, a frequência utilizada foi desigual. 0 fato demonstra que esse parâmetro parece não ser determinante para a efetividade do ultrassom no tratamento da tendinite calcária do ombro, que se caracteriza por uma patologia de caráter superficial.

A dose utilizada por Perron e Malouin (6) não é citada, inviabilizando qualquer tipo de decisão, e a falta de grupo controle no estudo de Greve et al. (25) intensifica a baixa qualidade do estudo.

Parâmetros do ultrassom terapêutico, como frequência, intensidade, regime de emissão (contínuo ou pulsado) empregado e tempo de aplicação, foram controversos entre os autores $(6,15,24,25)$ (Tabela $1)$ e interferem nos resultados $(43,44)$. Tanto o estudo de Ebenbichler et al. (15) quanto o de Shomoto et al. (24), além de terem registrado a efetividade do ultrassom terapêutico na tendinite calcária do ombro, apresentaram melhor qualidade metodológica em relação aos demais artigos. Conforme observado nos estudos revisados, os benefícios parecem estar relacionados à dose total e também à qualidade metodológica, que é um fator determinante para haver efetividade no tratamento da patologia. A randomização da amostra pode ser um fator importante para a distribuição igualitária entre os grupos de fatores prognósticos que também poderiam interferir nos resultados do estudo, sendo esse fato inserido na questão da qualidade metodológica.

Por meio da análise dos artigos desta revisão, sugere-se que o ultrassom terapêutico possa ser efetivo na diminuição ou resolução da tendinite calcária do ombro, bem como da sintomatologia presente nessa afecção, desde que haja adequada seleção de parâmetros para sua aplicação. Parece que a efetividade do ultrassom na tendinite calcária do ombro está relacionada à dose e ao número de sessões totais, ou seja, a quantidade de energia fornecida ao paciente.

\section{Conclusão}

Esta revisão sugere que o ultrassom pode ser um recurso importante no tratamento da tendinite calcária do ombro, podendo atuar positivamente na redução dos depósitos calcários desde que o recurso seja utilizado dentro de parâmetros satisfatórios. Para isso, a dose total, considerando-se a dose fornecida pelo recurso e o número de sessões, deve ser adequada ao objetivo do tratamento. Nesse 
sentido, faz-se necessária uma adequação metodológica para publicação de estudos que utilizem o ultrassom como recurso terapêutico no tratamento da tendinite calcária do ombro.

\section{Referências}

1. Unthoff HK. Calcifying tendinitis. Ann Chir Gynaecol. 1996;85(2):111-5.

2. Speed CA, Hazleman BL. Calcific tendinitis of the shoulder. N Engl J Med. 1999;340(20):1582-4.

3. Godinho GG, Freitas JMA, Vieira AW, Antunes LC, Castanheira EW. Tratamento artroscópico da tendinite calcária do ombro. Rev Bras Ortop. 1997;32(9): 669-74.

4. Wageck JPZ, Fernandez JS, Bender FL, Gervini FM. Tendinite calcárea do ombro. Acta med (Porto Alegre). 2007;28:270-9.

5. Bosworth BM. Calcium deposits in shoulder and subacromial bursitis: survey of shoulders. JAMA. 1941;116(22):2477-82.

6. Perron M, Malouin F. Acetic acid iontophoresis and ultrasound for the treatment of calcifying tendinitis of the shoulder: a randomized control trial. Arch Phys Med Rehabil. 1997;78(4):379-84.

7. Uhthoff HK, Loehr JF. Calcifying tendinitis. In: Rockwood A, Matsen FA, editores. The shoulder. Philadelphia, PA: Saunders; 1998.

8. Rathbun JB, Macnab I. The microvascular pattern of the rotator cuff. J Bone Joint Surg Br. 1970;52(3):540-53.

9. Booth REJ, Marvel JR. Differential diagnosis of shoulder pain. Orthop Clin North Am. 1975;6(2):353-79.

10. Charol MD, Cawston TE, Riley GP, Gresham GA, Hazleman BL. Rotator cuff degeneration and lateral epicondylitis: a comparative histological study. Ann Rheum Dis. 1994;53(1):30-4.

11. Yokoyama M, Aono H, Takeda A, Morita K. Cimetidine for chronic calcifying tendinitis of the shoulder. Reg Anesth Pain Med. 2003;28(3):248-52.

12. Pfister J, Gerber H. Chronic calcifying tendinitis of the shoulder - therapy by percutaneous needle aspiration and lavage: a prospective open study of 62 shoulders. Clin Rheumatol. 1997;16(3):269-74.
13. Uhthoff HK, Sarkar K. Calcifying tendinitis. In: Rockwood CA Jr, Matsen FA. The shoulder. Philadelphia, W.B.: Saunders; 1990.

14. Rompe JD, Zoellner J, Nafe B. Shock wave therapy versus conventional surgery in the treatment of calcifying tendinitis of the shoulder. Clin Orthop Relat Res. 2001;(387):72-82.

15. Ebenbichler GR, Erdogmus CB, Resch CL, Funovics MA, Kainberger F, Barisani G et al. Ultrasound therapy for calcific tendinitis of the shoulder. $\mathrm{N}$ Engl J Med. 1999;340(20):1533-8.

16. Ark JW, Flock TJ, Flatow EL, Bigliani LU. Arthroscopic treatment of calcific tendinitis of the shoulder. Arthroscopy. 1992;8(2):183-8.

17. Gam NA, Johannsen NF. Ultrasound therapy in musculoskeletal disorders: a meta-analylis. Pain. 1995;63(1):85-91.

18. Van der Windt DA, van der Heijdeb GJ, van der Berg SG, Ter Riet G, de Winter AF, Bouter LM. Ultrasound therapy for musculoskeletal disorders: a systematic review. Pain. 1999;81(3):257-71.

19. Robertson VJ, Baker KG. A review of therapeutic ultrasound: effectiveness studies. Phys Ther. 2001; 81(7):1339-50.

20. Toro JR, Monje MR, Puentes EC, Rebollo AG, Sanchez EB. Tratamiento de la tendinitis calcificante del hombro mediante iontoforesis con ácido acético y ultrasonidos. Rehabilitación (Madri). 2001;35(3):166-70.

21. Leduc BE, Caya J, Tremblay S, Bureau NJ, Dumont M. Treatment of calcifying tendinitis of the shoulder by acetic acid iontophoresis: a double-blind randomized controlled trial. Arch Arch Phys Med Rehabil. 2003;84(10):1523-7.

22. Jadad AR, Moore RA, Carroll D, Jenkinson C, Reynolds DJ, Gavaghan DJ et al. Assessing the quality of reports of randomized clinical trials: is blinding necessary? Control Clin Trials. 1996;17(1):1-12.

23. Mcneely ML, Olivo SA, Magee DJ. A systematic review of the effectiveness of physical therapy interventions for temporomandibular disorders. Phys Ther. 2006;86(5):710-25.

24. Shomoto S, Takatori K, Morishita S, Nagino K, Yamamoto W, Shimohira $\mathrm{T}$ et al. Effects of ultrasound therapy of calcificated tendinitis of the shoulder. J Jpn Phys Ther Assoc. 2002;5:7-11. 
25. Greve JMD, Rossi J, Cossermelli W, Ferreira Filho A, Castro AW, Algranti C. Reabilitação funcional das lesões tendinosas degenerativas do ombro. Rev Hosp Clin Fac Med Univ São Paulo. 1991;46(2):78-81.

26. Durigan JLQ, Baretta IP, Costa CN, Borges HE. Efeitos do ultra-som terapêutico pulsado associado a indometacina no edema de ratos artríticos. Fisioter Bras. 2005;6(2):130-5.

27. Zanon RG, Brasil AK, Imamoura M. Ultra-som contínuo no tratamento da fasciíte plantar crônica. Acta Ortop. 2006;14(3):137-40.

28. Fontoura HS, Azevedo RB. Influência das propriedades antiinflamatórias do ultra-som terapêutico no tratamento de lesões musculares. Fisioter Bras. 2008;9(1):17-22.

29. Ter Haar G, Dyson M, Oakley EM. The use of ultrasound by physiotherapists in Britain. Ultrasound Med Biol. 1987;13(10):659-63.

30. Dyson M. Non-thermal cellular effects of ultrasound. Br J Cancer Suppl. 1982;5:165-71.

31. Kitchen SS, Partridge CJ. A review of therapeutic ultrasound. Physiotherapy. 1990;76:593-600.

32. Parizotto NA, Koeke PU, Moreno BG, Lourencin FTC. Utilização da fonoforese em desordens músculo-esqueléticas: uma meta-análise. Rev Bras Fisioter. 2003;7(1):9-15.

33. Guirro ECO, Guirro R, Ferreira AL. Efeitos da estimulação ultrasônica pulsada de baixa intensidade no processo cicatricial: estudo experimental em ratos. Revista Cienc Tecnol. 2005;2(8):37-47.

34. Dyson M. Mechanisms involved in therapeutic ultrasound. Physiotherapy. 1982;79(3):116-20.

35. Lehmann JF. Ultrasom therapy in therapeutic heat and cold. Baltimore: Williams \& Wilkins; 1990.

36. Dinno MA, Crum LA, Wu J. The effect of therapeutic ultrasound on electrophysiological parameters of frog skin. Ultrasound Med Biol. 1989;5(5):461-70.

37. Dyson M, Suckling J. Stimulation of tissue repair by ultrasound: a survey of the mechanisms involved. Physiotherapy. 1978;64(4):105-8.

38. Roche C, West JA. Controlled trial investigating the effect of ultrasound on venous ulcers referred from general practitioners. Physiotherapy. 1984;70:475-7.
39. Pospisilová J. Effect of ultrasound on collagen synthesis and deposition in experimental granuloma tissue. Possibilities of clinical use of ultrasound in healing disorders. Acta Chir Plast. 1976;18(4):176-83.

40. Kopakkala-Tani M, Leskinen JJ, Karjalainen HM, Karjalainen T, Hynynen K, Töyräs J, et al. Ultrasound stimulates proteoglycan synthesis in bovine primary chondrocytes. Biorheology. 2006;43(3-4):271-82.

41. Fyfe MC, Chah LA. Mast cell degranulation: a possible mechanism of action of therapeutic ultrasound. Ultrasound Med Biol. 1982;8(Suppl 1):62.

42. Young SR, Dyson M. Macrophage responsiveness to therapeutic ultrasound. Ultrasound Med Biol. 1990;16(8):809-16.

43. Baker KG, Robertson VJ, Duck FA. A review of therapeutic ultrasound: biophysical effects. Phys Ther. 2001;81(7):1351-8.

44. Robertson VJ. Dosage and treatment response in randomized clinical trials of therapeutic ultrasound. Phys Ther Sport. 2002;3:124-33.

45. Draper D0, Castel JC, Castel D. Rate of temperature increase in human muscle during $1 \mathrm{MHz}$ and $3 \mathrm{MHz}$ continuous ultrasound. J Orthop Sports Phys Ther. 1995;22(4):142-50.

46. Starkey C. Recursos terapêuticos em fisioterapia. São Paulo: Manole; 2001.

47. Low J, Reed A. Ultra som terapêutico. In: Low J, Reed A. Eletroterapia explicada: princípios e prática. 3a ed. Barueri: Manole; 2001.

48. Maggi LE, Omena TP, von Krüger MA, Pereira WCA. Software didático para modelagem do padrão de aquecimento dos tecidos irradiados por ultra-som fisioterapêutico. Rev Bras Fisioter. 2008; 12(3):204-14.

Recebido: $16 / 02 / 2011$

Received: 02/16/2011

Aprovado: $15 / 07 / 2011$

Approved: 07/15/2011 